\title{
Identification of an anti-plant-virus molecule in Alpinia zerumbet
}

\author{
Tadashi Hatanaka ${ }^{1 *+} \mathbb{0}$, Mari Narusaka', Misugi Uraji ${ }^{1,2}$, Yasuyuki Yamaji ${ }^{3}$ and Yoshihiro Narusaka ${ }^{1 \dagger}$
}

\begin{abstract}
In plants, viral diseases are second only to fungal diseases in terms of occurrence, and cause substantial damage to agricultural crops. The aqueous extracts of shell ginger, Alpinia zerumbet exhibit inhibitory effects against virus infections in belonging to the Solanaceae family. In this study, we isolated an anti-plant-virus molecule from the extracts using a conventional method involving a combination of reversed phase column chromatography, dialysis, and lyophilization. The anti-plant-virus molecule was identified as proanthocyanidin, which mostly consisted of epicatechin and exhibited more than 40 degrees of polymerization.
\end{abstract}

Keywords: Alpinia zerumbet, Shell ginger, Proanthocyanidin, Anti-plant-virus molecule

\section{Introduction}

Recent advances in biotechnology and chemistry of natural products have enabled to make substantial progress in bio-pesticide development. Bio-pesticides have garnered considerable interest in recent years because they are environmentally safe, owing to the fact that they decompose quickly and leave little residue. The production of bio-pesticides is currently growing at a rate of $16 \%$ per year, which is approximately three-times higher than that of conventional agrochemicals (5.5\% per year, Marrone 2013).

In plants, viral diseases are second only to fungal diseases in terms of occurrence, causing substantial damage to agricultural crops. Globally, plant viruses cause economic losses of approximately $\$ 60$ billion annually, and the loss of food crops alone account for $\$ 20$ billion per year (Xie et al. 2009).

It is well known that plant viruses are difficult to control compared to other plant pathogens primarily due to the lack of chemicals that are effective against plant

\footnotetext{
*Correspondence: hatanaka@bio-ribs.com

†Tadashi Hatanaka and Yoshihiro Narusaka contributed equally to this study

${ }^{1}$ Okayama Prefectural Technology Center for Agriculture, Forestry, and Fisheries, Research Institute for Biological Sciences (RIBS), 7549-1

Kibichuo-cho, Kaga-gun, Okayama 716-1241, Japan

Full list of author information is available at the end of the article
}

viruses. To limit the damage caused by plant viruses, different management strategies have been applied, including prevention of plant virus spread by limiting the spread of transmitting vectors using insecticides, breeding cultivars that are resistant to virus infection, or simply cutting down infected plants. However, these efforts are still limited, and are not effective enough. Researchers have attempted to identify and develop anti-plant-virus agents, and recently, significant progress has been made in the field of biogenic anti-plant-virus substances (Zhao et al. 2017). Biogenic anti-plant-virus substances include proteins, polysaccharides, and small molecules from plants, microorganisms, algae, and animals. However, the number of anti-plant-virus substances with high efficiency and good economic benefits is still limited (Zhao et al. 2017).

More than 500 compounds have been isolated from 35 Alpinia species, and the major compounds are terpenoids and diarylheptanoids. The crude extracts and identified compounds exhibit a broad spectrum of bioactivities including anti-emetic, anti-ulcer, anti-bacterial, anti-inflammatory, anti-amnesic, and anti-cancer activities (Ma et al. 2017). Shell ginger, A. zerumbet (Pers.) B.L. Burtt \& R.M. Smith, a member of the Zingiberaceae family (Teschke and Xuan 2018), grows widely in subtropical and tropical regions in East Asia, including the Okinawa Islands in Japan. A. zerumbet is commonly used 
in traditional Okinawa cuisine and as a herbal medicine. Bioactive compounds have been isolated and identified from crude $A$. zerumbet extracts (Tawata et al. 2008). A recent study demonstrated that $A$. zerumbet substantially increased the lifespan of Caenorhabditis elegans (Upadhyay et al. 2013).

In our previous study, we found that aqueous extracts of $A$. zerumbet exerted inhibitory effects against virus infection in plants belonging to the Solanaceae family (Narusaka et al. 2020).

In the present study, we isolated and identified an antiplant-virus molecule, proanthocyanidin (PAC), in $A$. zerumbet.

\section{Materials and methods Chemicals}

Procyanidins A2 and B1 were purchased from Funakoshi (Tokyo, Japan). 4-Dimethylaminocinnamaldehyde (DMAC) was obtained from Sigma-Aldrich (Tokyo, Japan).

\section{Plant material}

We collected A. zerumbet plants, known as "Gettou" or "Shima-gettou", in Nakijin Village, Okinawa Prefecture, Japan, and used the extracts of these plants to identify anti-plant-viral molecules.

\section{Purification of the anti-plant-viral molecule}

An aqueous extract of $A$. zerumbet was obtained by squeezing the leaves and stems using a sugar cane squeezer (YBK-2, Yabiku, Okinawa, Japan). The extract was centrifuged $(3260 \times g)$ for $10 \mathrm{~min}$, and the supernatant was filtered to remove debris. Filtrate $(100 \mathrm{~mL})$ was applied to a C18 Sep-Pak ${ }^{\circledR}$ cartridge $(10 \mathrm{~g}(35 \mathrm{cc})$; Nihon-Waters, Tokyo, Japan), which was equilibrated with distilled water before use. Thereafter, the column was washed with $100 \mathrm{~mL}$ of distilled water and eluted with $100 \mathrm{~mL}$ of $5 \%, 10 \%, 20 \%$, and $40 \%$ acetonitrile. The fraction rich in anti-plant-viral molecules was obtained by elution with $100 \mathrm{~mL}$ of $20 \%$ acetonitrile. The fraction was evaporated to remove acetonitrile under reduced pressure at $50{ }^{\circ} \mathrm{C}$, and the resultant solution was dialyzed using a dialysis membrane (cutoff $10 \mathrm{kDa}$ ) against distilled water ( $4 \mathrm{~L} \times 4$ times), and the dialysate was lyophilized. The resultant powder was used to characterize the purified anti-plant-viral molecule. The anti-plantviral molecule was characterized by matrix-assisted laser desorption/ionization time of flight (MALDI-TOF) mass spectrometry (MS), ${ }^{13} \mathrm{C}$ nuclear magnetic resonance (NMR) analysis, gel permeation chromatography (GPC), and spectrophotometric assays using DMAC.

\section{Antiviral assay}

The antiviral assay was performed following a method described in our previous study (Narusaka et al. 2020). To evaluate the inhibitory effect of purified sample on viruses, the sample solution was applied on Nicotiana benthamiana plants (in the third true leaf stage) as foliar sprays only once; three days post treatment, the plants were inoculated with tomato mosaic virus (ToMV). Inoculation of $N$. benthamiana plants with ToMV was performed as described, with some modifications, including using pTL-derived plasmids (pTLBN. G3), which contain a full-length ToMV cDNA and a gene encoding green fluorescent protein (GFP) (Kubota et al. 2003). In vitro transcription of $2 \mu \mathrm{g}$ of the template DNA using the AmpliCap-Max T7 High Yield Message Maker Kit (CELLSCRIPT, Madison, WI, USA) was performed at $37^{\circ} \mathrm{C}$ for $40 \mathrm{~min}$ in a $20-\mu \mathrm{L}$ reaction. Third true leaves of $N$. benthamiana that were treated with either the sample solution or distilled water (controls) were mechanically inoculated with $20 \mu \mathrm{L}$ of 40 -fold dilution of the transcription mixture. GFP foci were used to detect virus infection, and they were observed under blue light irradiation at 3 days post-inoculation (dpi).

The antiviral activity was assessed based on the number of GFP foci formed on the control and the treated $N$. benthamiana leaves. Statistical analyses were performed using one-way analysis of variance (ANOVA) with the Tukey-Kramer method.

\section{MALDI-TOF-MS analysis}

The purified sample $(1 \mathrm{mg})$ was dissolved in $1 \mathrm{~mL}$ of distilled water. 2,5-dihydroxy benzoic acid was used as a matrix in the positive-ion mode. To enhance ion formation, sodium chloride was added to the matrix. A MALDI-TOF-MS spectrum was acquired using a Shimadzu MALDI-8020 MALDI-TOF-MS apparatus (Shimadzu, Kyoto, Japan).

\section{DMAC assay}

We performed DMAC assays as previously described by Wang (Wang et al. 2016). A methanol-based DMAC reagent was prepared by adding $50 \mathrm{mg}$ of DMAC to $17.5 \mathrm{~mL}$ of hydrochloric acid (37\%), and then making up the volume to $50 \mathrm{~mL}$ with methanol. The purified sample stock solution $(2.5 \mathrm{mg} / \mathrm{mL})$ was diluted with methanol to $0.25 \mathrm{mg} / \mathrm{mL}$. A mixture of $0.125 \mathrm{~mL}$ of sample solution and $0.875 \mathrm{~mL}$ of DMAC reagent was prepared in cuvettes, with a $1-\mathrm{cm}$ pass length. The spectrum (400$700 \mathrm{~nm}$ ) of the DMAC conjugate was measured using an $\mathrm{SH}-8000$ spectrometer (CORONA Electric, Hitachi, Japan). 


\section{GPC analysis}

The GPC analysis was performed at Toray Research Center (Tokyo, Japan). The purified sample (1 mg) was dissolved in $1 \mathrm{~mL}$ of dimethyl formamide (DMF) containing $50 \mathrm{mM}$ lithium chloride $(\mathrm{LiCl})$, and $200 \mu \mathrm{L}$ of the sample was subjected to GPC. The molecular mass was estimated using a high-performance liquid chromatograph equipped with a refractive index detector (RI-8020; TOSOH, Tokyo, Japan), and TSK gel $\alpha-4000$ and TSK gel $\alpha-2500$ columns $(7.8 \mathrm{~mm} \times 30 \mathrm{~cm}$; TOSOH, Tokyo, Japan), which were connected directly. DMF containing $50 \mathrm{mM} \mathrm{LiCl}$ was used as the solvent at a flow rate of $0.7 \mathrm{~mL} / \mathrm{min}$ and the column temperature was maintained at $23{ }^{\circ} \mathrm{C}$. The eluted sample was used for molecular mass determination, with a calibration curve obtained using standard polystyrene kits (PSt Quick E and F; TOSOH, Tokyo, Japan).

\section{${ }^{13}$ C-NMR analysis}

We used a Varian VNMRS (600 MHz) spectrometer (Varian, Palo Alto, CA, USA) for the analysis. The purified sample was dissolved in acetone- $\mathrm{d} 6 / \mathrm{D}_{2} \mathrm{O}$ at a ratio of $1: 1$.

\section{Results and discussion}

\section{Purification of an antiviral molecule in A. zerumbet}

When the aqueous extract of $A$. zerumbet was fractioned using a reverse-phase chromatography and an antiviral assay was performed (Fig. 1), we found that the antiviral molecule was concentrated in the fraction obtained by elution with $20 \%$ acetonitrile. The fraction was then evaporated, dialyzed, and lyophilized. The resultant sample was used as the purified sample. The antiviral activity of the purified sample was confirmed by the inhibition against growth of GFP-tagged ToMV (Fig. 2). The protection value (\%) of the purified sample increased as the final concentration of the sample applied to the leaves increased. Thus, we successfully purified an antiplant-virus molecule from the aqueous extracts of $A$. zerumbet using a conventional method. To estimate the material balance in each fraction in the purification process, aliquots of each fraction were lyophilized (Table 1). The yield of the final fraction of the water extract was approximately $12 \%(\mathrm{w} / \mathrm{v})$. This fraction was rich in the active molecule, accounting for ca. $10 \%(\mathrm{w} / \mathrm{v})$ of the water extract of $A$. zerumbet.

\section{MALDI-TOF-MS analysis}

As shown in Fig. 3, a series of peaks with ions corresponding to sodium adducts $\left[\mathrm{M}+\mathrm{Na}^{+}\right]$distributed from $\mathrm{m} / \mathrm{z} 889.5$ to 6089.6 were observed in the mass spectrum of the purified sample obtained by
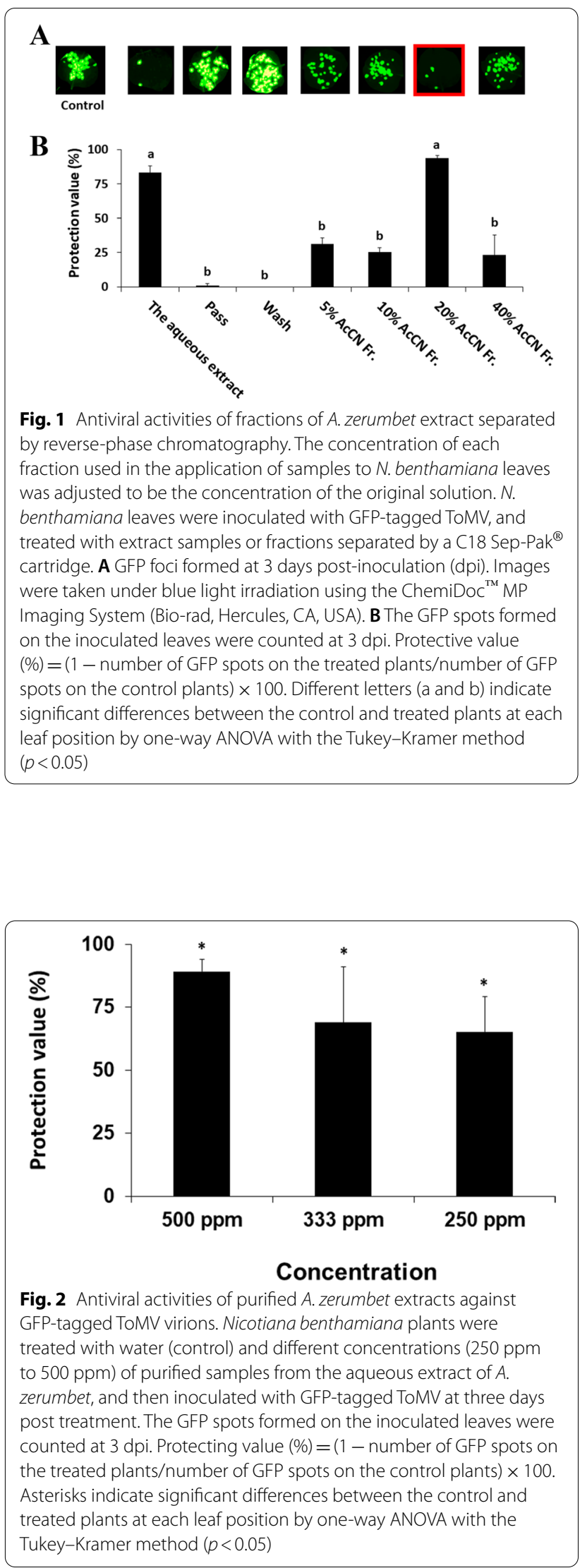


\begin{tabular}{|c|c|c|}
\hline Fraction & $\begin{array}{l}\text { Lyophilized material } \\
\text { (mg) }\end{array}$ & Yield (\%) \\
\hline Water extracts (100 mL) & 4321 & 100.0 \\
\hline Pass and wash & 3332 & 77.1 \\
\hline $20 \%$ acetonitrile eluate & 989 & 22.9 \\
\hline Dialysate & 504 & 11.7 \\
\hline
\end{tabular}

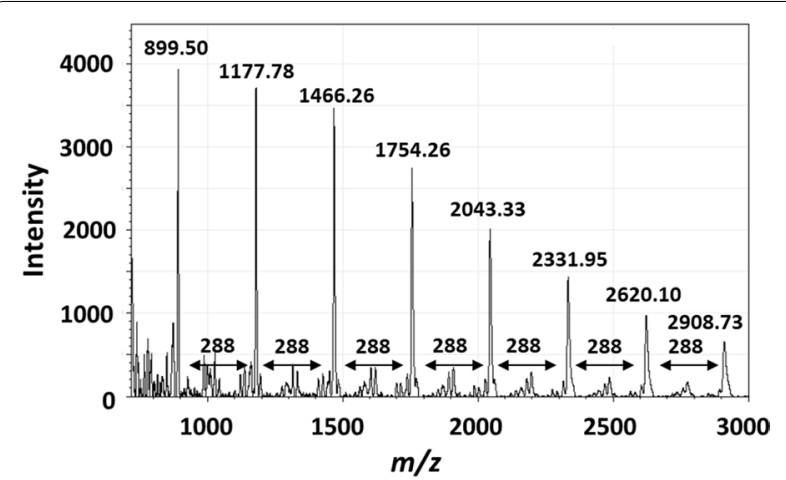

Fig. 3 MALDI-TOF MS spectrum of the purified aqueous extract of $A$. zerumbet

MALDI-TOF-MS. The distance between the adjacent ion peaks was $288 \mathrm{Da}\left(-\mathrm{C}_{15} \mathrm{H}_{12} \mathrm{O}_{6}-\right)$, corresponding to one catechin or epicatechin monomer. The results indicate that the active molecule is a polymer consisting of catechin or epicatechin. The mass intensity of the sample decreased with increasing degrees of polymerization (DP). The lower sensitivity of the large ions may be due to collisional fragmentations during the time of flight or when the ions are extracted from the matrix (Flamini 2003; Perret et al. 2003). MALDITOF-MS analysis enables the determination of the absolute molecular weight of individual chains with high accuracy, as long as the polymer is not too large (approximately $<10 \mathrm{kDa}$ ) (Koster et al. 2000). If intact molecular adducts are produced in the gas phase and the adducts are clearly identified, the analysis allows the confirmation of the nature of end-groups based on the sum of mass of adducted monomer units and both endgroups (Li et al. 2010; Charles 2014). From the data of the series of peaks of sodium adducts, we estimated the end structure of PAC. As shown in Table 2, both end structures were protons because the average molecular mass of both end-groups was 3.3 (ca. 2.0). Thus, the antiviral molecule in $A$. zerumbet is a PAC polymer, consisting of a simple condensation of catechin or epicatechin monomers.

\section{DMAC assay}

The polymeric nature of PACs causes in structural variations, including variations in the DP, linkage type, and position between constituent units. Catechin and epicatechin are the two most common flavan-3-ol units present in PAC polymers. The most common linkage of the flavan-3-ol units is a single $\mathrm{C}-\mathrm{C}$ bond (B-type), between the $\mathrm{C} 4$ of one flavan-3-ol unit (referred to as upper) and C8 or C6 of another (lower) unit. Wang et al. reported that in the absorption spectra, B-type dimers including procyanidin B1 showed a secondary 440- $\mathrm{nm}$ absorption peak, in addition to the primary 640-nm peak, which is typically measured using the DMAC assay (Wang et al. 2016). The A-type dimmers, with double interflavan linkage, showed only a $640-\mathrm{nm}$ absorbance peak in the assay (Fig. 4a). PAC in A. zerumbet-DMAC conjugate also showed a secondary 440-nm peak, as shown in Fig. 4b. Wang et al. have described that all B-type cocoa PACs exhibited a secondary 440$\mathrm{nm}$ absorbance peak that declined in intensity when DP increased (Wang et al. 2016). Based on these results, the PAC from A. zerumbet was categorized as a B-type.

Table 2 Estimation of end structures of PAC from A. zerumbet

\begin{tabular}{|c|c|c|c|c|c|}
\hline $\begin{array}{l}\text { Observed } \\
\text { molecular mass (A) }\end{array}$ & $\begin{array}{l}\text { Cation }\left(\mathrm{Na}^{+}\right) \\
\text {molecular mass (B) }\end{array}$ & $\begin{array}{l}\text { Exact molecular mass } \\
(\mathrm{A})-(\mathrm{B})=(\mathrm{C})\end{array}$ & $\begin{array}{l}\text { Unit molecular } \\
\text { mass (D) }\end{array}$ & $\begin{array}{l}\text { Degree } \\
\text { of polymerization } \\
(C) \div(D)=(E)\end{array}$ & $\begin{array}{l}\text { Estimated end } \\
\text { molecular masses } \\
(\mathrm{C})-((\mathrm{D}) \times(\mathrm{E}))\end{array}$ \\
\hline 889.50 & 22.99 & 866.51 & 288.06 & 3 & 2.3 \\
\hline 1177.78 & 22.99 & 1154.79 & 288.06 & 4 & 2.6 \\
\hline 1466.26 & 22.99 & 1443.27 & 288.06 & 5 & 3.0 \\
\hline 1754.55 & 22.99 & 1731.56 & 288.06 & 6 & 3.2 \\
\hline 2043.43 & 22.99 & 2020.44 & 288.06 & 7 & 4.0 \\
\hline \multirow[t]{3}{*}{2332.06} & 22.99 & 2309.07 & 288.06 & 8 & 4.6 \\
\hline & & & & & Average \\
\hline & & & & & 3.3 \\
\hline
\end{tabular}




\section{a}

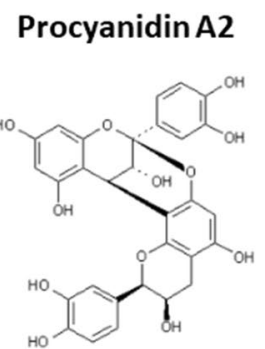

Procyanidin A2

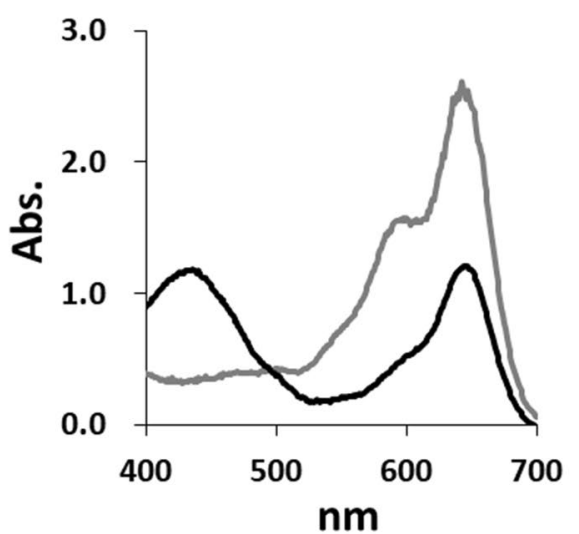

b

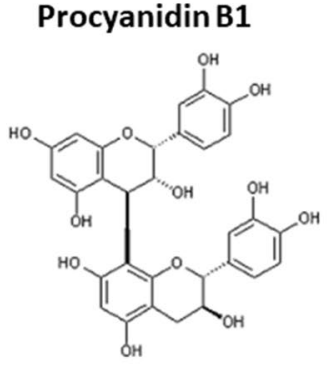

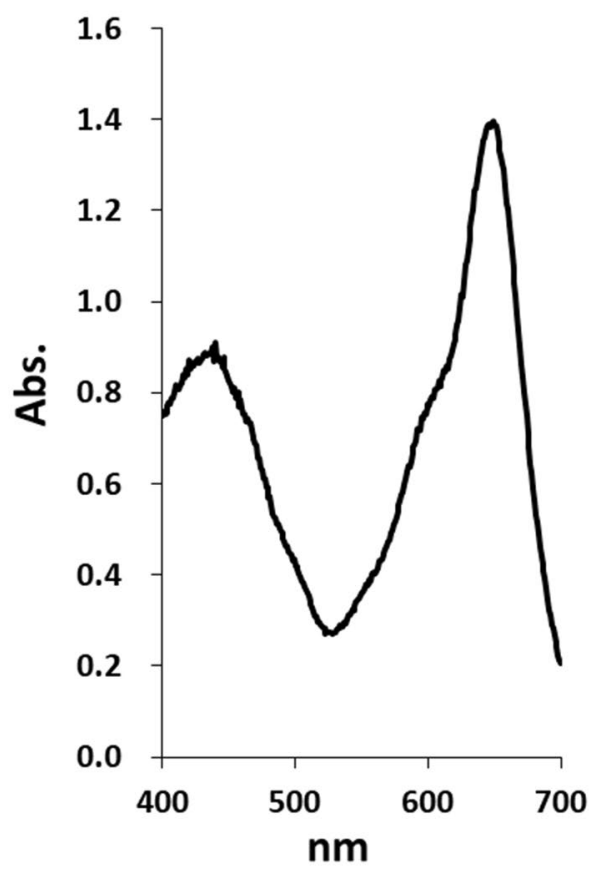

Fig. 4 Visible spectra of procyanidins and the purified sample from A. zerumbet aqueous extracts. a Chemical structure of procyanidins A2 and B1 (upper), the spectra of procyanidin A2 (gray line) and procyanidin B1 (black line), determined using the DMAC assay (lower). b The spectrum of the purified sample from the aqueous extract of $A$. zerumbet, obtained using the assay

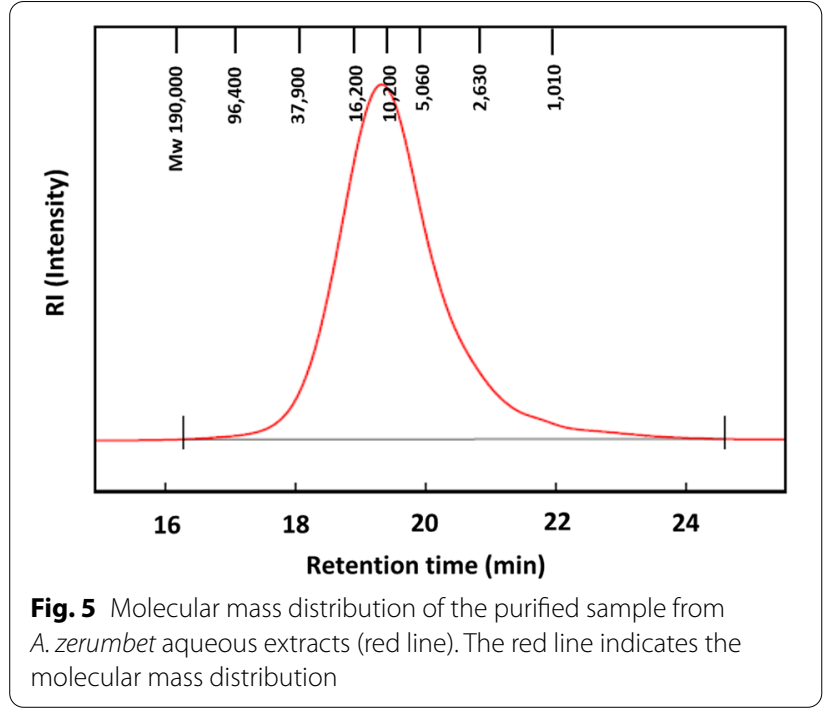

\section{GPC analysis}

Figure 5 shows the molecular mass of PAC from $A$. zerumbet. The weight-average molecular weight $(\mathrm{Mw})$, number average molecular weight $(\mathrm{Mn})$, polydispersity $(\mathrm{Mw} / \mathrm{Mn})$, and DP of the PAC were 12,400, 5,640, 2.2, and 43 , respectively.

\section{${ }^{13}$ C-NMR analysis}

The spectrum of PAC from A. zerumbet is shown in Fig. 5. Resonance assignments were conducted according to a previous study (Eberhardt and Young 1994). All assignments were consistent with those reported by Eberhardt and Young (1994). With respect to the aromatic B-ring attached at $\mathrm{C}-2$, the orientation of the hydroxyl group at $\mathrm{C}-3$ can be either cis or trans as shown in Fig. 6. The relative amounts of the two stereo-chemical configurations (2,3-cis or trans) in PAC from A. zerumbet were estimated from ${ }^{13} \mathrm{C}$-NMR spectra by integrating the resonances at 76.0 and $82.5 \mathrm{ppm}$ corresponding to $\mathrm{C}-2$ in 2,3-cis and 2,3-trans chain extender units, respectively. The results revealed that PAC in $A$. zerumbet almost consists of epicatechin (i.e., 2,3-cis configuration) because there was a low-intensity signal at $82.5 \mathrm{ppm}$, which was assigned a 2,3-trans configuration (Eberhardt and Young 1994). We observed several peaks between 68 and $80 \mathrm{ppm}$ (Fig. 6) that could not be assigned, and these peaks may have been derived from impurities.

To the best of our knowledge, the present study is the first to report the identification of an anti-plant-virus molecule, PAC, in A. zerumbet. PACs are synthesized as polymeric end-products of flavan-3-ols (catechin or epicatechin), which are biosynthesized from phenylalanine 


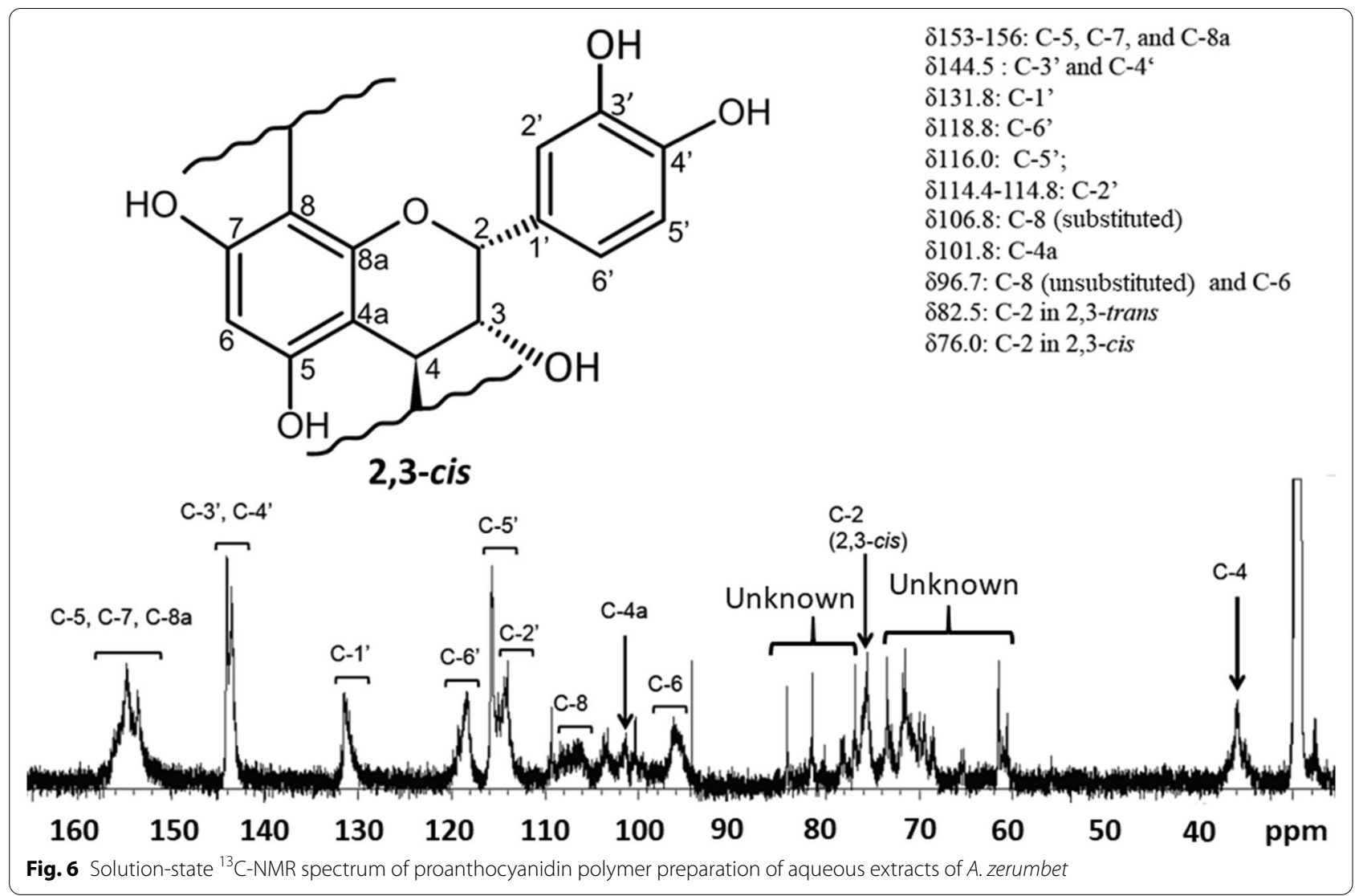

and malonyl-CoA via the flavonoid pathway. Phenylalanine is derived from the shikimate pathway, and malonyl-CoA is obtained from citrate that is produced by the tricarboxylic acid cycle (Oliveira et al. 2013). However, the polymerization pathway of PACs remains unexplained. We are currently attempting to analyze the genome of $A$. zerumbet to elucidate biosynthesis pathway of the PAC described in this study. Further studies are necessary to determine the mechanism of anti-plantvirus activity of PAC.

\section{Conclusions}

In this study, we identified the anti-plant-virus molecule in A. zerumbet as a PAC by using Nicotiana benthamiana plants and GFP-fused ToMV. The PAC, as the active molecule, was present at high proportions in the $10 \%(\mathrm{w} / \mathrm{v})$ water extract. In addition, the PAC was categorized as B-type, with more than $40 \mathrm{DP}$, and almost consisted of epicatechin. Thus, $A$. zerumbet is a potential bioresource of pesticides against viral diseases in plants.

\section{Abbreviations}

DMAC: 4-Dimethylaminocinnamaldehyde; PAC: Proanthocyanidin; MALDITOF: Matrix-assisted laser desorption/ionization time of flight; MS: Mass; NMR:
Nuclear magnetic resonance; GPC: Gel permeation chromatography; ToMV: Tomato mosaic virus; GFP: Green fluorescent protein; dpi: Days post-inoculation; ANOVA: Analysis of variance; DMF: Dimethyl formamide; LiCl: Lithium chloride; DP: Degree of polymerization; Mw: Weight-average molecular weight; Mn: Number average molecular weight.

\section{Acknowledgements}

We would like to thank Prof. Teruhiko Nitoda (Laboratory of Bioresources Chemistry, Graduate School of Environmental and Life Science, Okayama University, Japan) for providing suggestions on the ${ }^{13} \mathrm{C}-\mathrm{NMR}$ analysis. We also thank Dr. Masayuki Ishikawa (National Institute of Agrobiological Sciences, Japan) for providing the pTLBN. G3 plasmid used in this study.

\section{Authors' contributions \\ $\mathrm{MU}$ and TH purified the anti-plant-virus molecule from Alpinia zerumbet. YY, $\mathrm{YN}$, and MN conducted the antiviral assays. TH identified the anti-plant-virus molecule and wrote the manuscript. All authors read and approved the final manuscript.}

\section{Funding}

This research was supported by grants from the Science and Technology Research Promotion Program for Agriculture, Forestry, Fisheries and Food Industry, and the Project of the NARO Bio-oriented Technology Research Advancement Institution (Research Program on Development of Innovative Technology).

Availability of data and materials

All data generated or analyzed during this study are included in this published article.

Ethics approval and consent to participate Not applicable. 


\section{Consent for publication}

Not applicable.

\section{Competing interests}

The authors declare that they have no competing interests.

\section{Author details}

${ }^{1}$ Okayama Prefectural Technology Center for Agriculture, Forestry, and Fisheries, Research Institute for Biological Sciences (RIBS), 7549-1 Kibichuo-cho, Kaga-gun, Okayama 716-1241, Japan. ${ }^{2}$ Present Address: Graduate School of Science, Technology and Innovation, Kobe University, 1-1, Rokkodai-cho, Nada-ku, Kobe 657-8501, Japan. ${ }^{3}$ Department of Agricultural and Environmental Biology, Graduate School of Agricultural and Life Sciences, The University of Tokyo, 1-1-1, Yayoi, Bunkyo-ku, Tokyo 113-8657, Japan.

Received: 8 December 2020 Accepted: 11 February 2021

Published online: 21 February 2021

\section{References}

Charles L (2014) MALDI of synthetic polymers with labile end-groups. Mass Spectrom Rev 33:523-543

Eberhardt TL, Young RA (1994) Confer seed cone proanthocyanidin polymers: characterization by ${ }^{13} \mathrm{C}$ NMR spectroscopy and determination of antifungal activities. J Agric Food Chem 42:1704-1708

Flamini R (2003) Mass spectrometry in grape and wine chemistry. Part I: polyphenols. Mass Specrom Rev 22:218-250

Koster S, Duursma MC, Boon JJ et al (2000) Structural analysis of synthetic homo- and copolyesters by electrospray ionization on a Fourier transform ion cyclotron resonance mass spectrometer. J Mass Spectrom 35:739-748

Kubota K, Tsuda S, Tamai A, Meshi T (2003) Tomato mosaic virus replication protein suppresses virus-targetted posttranscriptional gene silencing. J Virol77:11016-11026
Li Y, Hoskins JN, Sreerama SG et al (2010) The identification of synthetic homopolymer end groups and verification of their transformations using MALDI-TOF mass spectrometry. J Mass Spectrom 45:587-611

Ma XN, Xie L, Miao YQ, Yang XW (2017) An overview of chemical constitutions from Alpinia species in the last six decades. RSC Adv 7:14114-14144

Marrone PG (2013) Market opportunities for bio-pesticides:104th National Meeting, Exposition, Picogram, American Chemical Society, p 246

Narusaka M, Yamaji Y, Uraji M et al (2020) Inhibitory effects of Alpinia zerumbet extract against plant virus infection in solanaceous plants. Plant Biotechnol 37:93-97

Oliveira J, Mateus N, Freitas V (2013) Flavanols: catechins and proanthocyanidins. In: Ranawat KG, Mérillon JM (eds) Natural products. Springer, Berlin

Perret C, Pezet R, Tabacchi R (2003) Fractionation of grape tannins and analysis by matrix-assisted laser desorption/ionisation time-of-flight mass spectrometry. Phytochem Anal 14:202-208

Tawata S, Fukuta M, Xuan TD, Deba F (2008) Total utilization of tropical plants Leucaena leucocephala and Alpinia zerumbet. J Pestic Sci 33:40-43

Teschke R, Xuan TD (2018) Viewpoint: A contributory role of shell ginger (Alpinia zerumbet) for human longevity in Okinawa, Japan? Nutrients 10:166

Upadhyay A, Chompoo J, Taira N et al (2013) Significant longevity-extending effects of Alpinia zerumbet leaf extracts on the life span of Caenorhabditis elegans. Biosci Biotechnol Biochem 60:1643-1645

Wang Y, Singh AP, Hurst WJ et al (2016) Influence of degree-of-polymerization and linkage on the quantification of proanthocyanidins using 4-dimethylaminocinnamaldehyde (DMAC) assay. J Agric Food Chem 64:2190-2199

Xie LH, Lin QY, Wu ZJ (2009) Plant virus: virology and molecular biology. Science Press, Beijing

Zhao L, Feng C, Wu K et al (2017) Advances and prospects in biogenic substances against plant virus: a review. Pestic Biochem Physiol 135:15-36

\section{Publisher's Note}

Springer Nature remains neutral with regard to jurisdictional claims in published maps and institutional affiliations.

\section{Submit your manuscript to a SpringerOpen ${ }^{\circ}$ journal and benefit from:}

- Convenient online submission

- Rigorous peer review

- Open access: articles freely available online

- High visibility within the field

- Retaining the copyright to your article

Submit your next manuscript at $\boldsymbol{\nabla}$ springeropen.com 